\title{
Grids of stellar models
}

\author{
VIII. From 0.4 to $1.0 M_{\odot}$ at $Z=0.020$ and $Z=0.001$, with the MHD equation of state \\ C. Charbonnel ${ }^{1,2}$, W. Däppen ${ }^{3}$, D. Schaerer ${ }^{1,2}$, P.A. Bernasconi ${ }^{4}$, A. Maeder ${ }^{4}$, G. Meynet ${ }^{4}$, and N. Mowlavi ${ }^{4}$ \\ 1 Laboratoire d'Astrophysique de Toulouse, CNRS UMR 5572, 14 Av. E. Belin, 31400 Toulouse, France \\ 2 Space Telescope Science Institute, 3700 San Martin Drive, Baltimore, MD 21218, U.S.A. \\ 3 Department of Physics and Astronomy, University of Southern California, Los Angeles, CA 90089-1342, U.S.A. \\ ${ }^{4}$ Geneva Observatory, CH-1290 Sauverny, Switzerland
}

Received March 13, 1997; accepted October 23, 1998

\begin{abstract}
We present stellar evolutionary models covering the mass range from 0.4 to $1 M_{\odot}$ calculated for metallicities $Z=0.020$ and 0.001 with the MHD equation of state (Hummer \& Mihalas 1988; Mihalas et al. 1988; Däppen et al. 1988). A parallel calculation using the OPAL (Rogers et al. 1996) equation of state has been made to demonstrate the adequacy of the MHD equation of state in the range of 1.0 to $0.8 M_{\odot}$ (the lower end of the OPAL tables). Below, down to $0.4 M_{\odot}$, we have justified the use of the MHD equation of state by theoretical arguments and the findings of Chabrier \& Baraffe (1997).

We use the radiative opacities by Iglesias \& Rogers (1996), completed with the atomic and molecular opacities by Alexander \& Fergusson (1994). We follow the evolution from the Hayashi fully convective configuration up to the red giant tip for the most massive stars, and up to an age of $20 \mathrm{Gyr}$ for the less massive ones. We compare our solarmetallicity models with recent models computed by other groups and with observations.

The present stellar models complete the set of grids computed with the same up-to-date input physics by the Geneva group $(Z=0.020$ and 0.001 , Schaller et al. 1992; Bernasconi 1996, and Charbonnel et al. 1996; $Z=0.008$, Schaerer et al. 1992; $Z=0.004$, Charbonnel et al. 1993; $Z=0.040$, Schaerer et al. 1993; $Z=0.10$, Mowlavi et al. 1998; enhanced mass loss rate evolutionary tracks, Meynet et al. 1994).
\end{abstract}

Key words: stars: evolution — stars: Hertzsprung-russell diagram — stars: interiors — stars: low-mass

Send offprint requests to: C. Charbonnel

* Data available at the CDS via anonymous ftp to cdsarc.u-strasbg.fr (130.79.128.5) or via http://cdsweb.ustrasbg.fr/Abstract.html

\section{Introduction}

In stellar evolution computations, and in particular in the case of stars more massive than the Sun, it is generally sufficient to use a simple equation of state. The plasma of the stellar interior is treated as a mixture of perfect gases of all species (atoms, ions, nuclei and electrons), and the Saha equation is solved to yield the degrees of ionization or molecular formation. In the case of low mass stars however, non ideal effects, such as Coulomb interactions become important. It is then necessary to use a more adequate equation of state than the one employed in the Geneva code for more massive stars. This simple equation of state essentially contains a mixture of ideal gases, ionization of the chemicals is dealt with by the Saha equation, excited states and molecules are neglected, complete pressure ionization is artificially imposed above certain temperatures and pressures, and no Coulomb-pressure correction is included (see Schaller et al. 1992).

For the present grids of models of 0.4 to $1.0 M_{\odot}$ stars these assumptions are obviously inadequate. For such stars, the most useful equations of state, as far as their smooth realization and versatility are concerned, are (i) the so-called Mihalas-Hummer-Däppen (MHD) equation of state (Hummer \& Mihalas 1988; Mihalas et al. 1988; Däppen et al. 1988), and (ii) the OPAL equation of state, the major alternative approach developed at Livermore (Rogers 1986, and references therein; Rogers et al. 1996). A brief description of these two equations of state is given in the next section.

Here, we chose the MHD equation of state. First, we were able to compute very smooth tables specifically for our cases of chemical composition, instead of relying on pre-computed, relatively coarse tables that would require interpolation in the chemical composition. Second, our choice was forced by the fact that the currently available OPAL equation of state tables do not allow to go 
below stars less massive than $\sim 0.8 M_{\odot}$. Third, we validated our choice by a comparative calculation with OPAL at its low-mass end. We found results that are virtually indistinguishable from MHD. Fourth, we examined in a parallel theoretical study (Trampedach \& Däppen 1998) the arguments about the validity of the MHD equation of state down to the limit of our calculation of $0.4 M_{\odot}$ (see below). Therefore we do not have to include a harder excluded-volume term such as the one included in the Saumon-Chabrier (SC) equation of state (Saumon \& Chabrier 1991, 1992).

Although the MHD equation of state was originally designed to provide the level populations for opacity calculations of stellar envelopes, the associated thermodynamic quantities of MHD can none the less be reliably used also for stellar cores. This is due to the fact that in the deeper interior the plasma becomes virtually fully ionized. Therefore, in practice, it does not matter that the condition to apply the detailed Hummer-Mihalas (1988) occupation formalism for bound species is not fulfilled, because essentially there are no bound species. Other than that, the MHD equation of state includes the usual Coulomb pressure and electron degeneracy, and can therefore be used for low-mass stars and, in principle, even for envelopes of white dwarfs (W. Stolzmann, private communication). The present paper, with its MHD-OPAL comparison (see Sect. 3) corroborates this assertion.

This broad applicability of the MHD equation of state for entire stars was specifically demonstrated by its successes in solar modeling and helioseismology (ChristensenDalsgaard et al. 1988; Charbonnel \& Lebreton 1993; Richard et al. 1996; Christensen-Dalsgaard et al. 1996). A solar model that is based on the MHD equation of state from the surface to the center is in all respects very similar to one based on the OPAL equation of state, the major alternative approach developed at Livermore (Rogers 1986, and references therein; Rogers et al. 1996). This similarity even pertains to the theoretical oscillation frequencies that are used in comparisons with the observed helioseismic data.

Although the difference between the MHD and OPAL equations of state is of helioseismological relevance, it has no importance for the lower-mass stellar modeling of the present analysis. This is explicitly validated in the present paper. For the much finer helioseismological analyses, it turned out that in some respect the OPAL model seems to be closer than the MHD model to the one inferred from helioseismological observations (ChristensenDalsgaard et al. 1996; Basu \& Christensen-Dalsgaard 1997). However, we stress that for the present stellar modelling these subtle differences are no compelling reason to abandon the convenience of our ability to compute MHD equation of state tables ourselves, and to go below the range of the available OPAL tables $\left(\sim 0.8 M_{\odot}\right)$.

Not only helioseismology, but also fine features in the Hertzsprung-Russell diagram of low- and very low-mass stars impose strong constraints on stellar models (Lebreton \& Däppen 1988; D'Antona \& Mazzitelli 1994, 1996; Baraffe et al. 1995; Saumon et al. 1995). They have all confirmed the validity of the principal equation-of-state ingredients employed in MHD (Coulomb pressure, partial degeneracy of electrons, pressure ionization). Finally, we have checked that even at the low-mass end of our calculations the physical mechanism for pressure ionization in the MHD equation of state is still achieved by the primary pressure ionization effect of MHD (the reduction of bound-state occupation probabilities due to the electrical microfield; see Hummer \& Mihalas 1988). Such a verification was necessary to be sure that our results are not contaminated by the secondary, artificial pressure-ionization device included in MHD for the very low-temperature high-density regime (the so-called $\Psi$ term of Mihalas et al. 1988). A parallel calculation has confirmed that in our models a contamination by this $\Psi$ term can be ruled out (Trampedach \& Däppen 1998).

In the present paper, we expand the current mass range of the Geneva evolution models from 0.8 down to $0.4 M_{\odot}$, by using a specifically calculated set of tables of the MHD equation of state. This work aims to complete the base of extensive grids of stellar models computed by the Geneva group with up-to-date input physics $(Z=$ 0.020 and 0.001, Schaller et al. (1992); Bernasconi (1996), and Charbonnel et al. (1996); $Z=0.008$, Schaerer et al. (1992); $Z=0.004$, Charbonnel et al. (1993); $Z=0.040$, Schaerer et al. (1993); $Z=0.10$, Mowlavi et al. (1998); enhanced mass loss rate evolutionary tracks, Meynet et al. (1994)). In Sect. 2, we present the characteristics of our equation of state and recall the physical ingredients used in our computations. In Sect. 3, we summarize the main characteristics of the present models and discuss the influence of the equation of state on the properties of low mass stars. Finally, we compare our solar-metallicity models with recent models computed by other groups and with observations in Sect. 4.

\section{Input physics}

The basic physical ingredients used for the complete set of grids of the Geneva group are extensively described in previous papers (see Schaller et al. 1992, hereafter Paper I). With the exception of the equation of state, described in detail in the following subsection, we therefore just mention the main points.

\subsection{Equation of state}

We have already justified our choice of the MHD equation of state in the introduction. As mentioned there, MHD is one of the two recent equations of state that have been especially successful in modeling the Sun under the 
strong constraint of helioseismological data. Historically, the MHD equation of state was developed as part of the international "Opacity Project" (OP, see Seaton 1987, 1992). It was realized in the so-called chemical picture, where plasma interactions are treated with modifications of atomic states, i.e. the quantum mechanical problem is solved before statistical mechanics is applied. It is based on the so-called free-energy minimization method. This method uses approximate statistical mechanical models (for example the nonrelativistic electron gas, DebyeHückel theory for ionic species, hard-core atoms to simulate pressure ionization via configurational terms, quantum mechanical models of atoms in perturbed fields, etc.). From these models a macroscopic free energy is constructed as a function of temperature $T$, volume $V$, and the concentrations $N_{1}, \ldots, N_{m}$ of the $m$ components of the plasma. The free energy is minimized subject to the stoichiometric constraint. The solution of this minimum problem then gives both the equilibrium concentrations and, if inserted in the free energy and its derivatives, the equation of state and the thermodynamic quantities.

The other of these two equations of state is the one underlying the OPAL opacity project (see Sect. 2.2). The OPAL equation of state is realized in the so-called physical picture. It starts out from the grand canonical ensemble of a system of the basic constituents (electrons and nuclei), interacting through the Coulomb potential. Configurations corresponding to bound combinations of electrons and nuclei, such as ions, atoms, and molecules, arise in this ensemble naturally as terms in cluster expansions. Any effects of the plasma environment on the internal states are obtained directly from the statisticalmechanical analysis, rather than by assertion as in the chemical picture.

More specifically, in the chemical picture, perturbed atoms must be introduced on a more-or-less ad-hoc basis to avoid the familiar divergence of internal partition functions (see e.g. Ebeling et al. 1976). In other words, the approximation of unperturbed atoms precludes the application of standard statistical mechanics, i.e. the attribution of a Boltzmann-factor to each atomic state. The conventional remedy of the chemical picture against this is a modification of the atomic states, e.g. by cutting off the highly excited states in function of density and temperature of the plasma. Such cut-offs, however, have in general dire consequences due to the discrete nature of the atomic spectrum, i.e. jumps in the number of excited states (and thus in the partition functions and in the free energy) despite smoothly varying external parameters (temperature and density). However, the occupation probability formalism employed by the MHD equation of state avoids these jumps and delivers very smooth thermodynamic quantities. Specifically, the essence of the MHD equation of state is the Hummer-Mihalas (1988) occupation probability formalism, which describes the reduced availability of bound systems immersed in a plasma. Perturbations by charged and neutral particles are taken into account. The neutral contribution is evaluated in a first-order approximation, which is good for stars in which most of the ionization in the interior is achieved by temperature (the aforementioned study (Trampedach \& Däppen 1998) has verified the validity of this assumption down to the lowest mass of our calculation). For colder objects (brown dwarfs, giant planets), higher-order excluded-volume effects become very important (Saumon \& Chabrier 1991, 1992; Saumon et al. 1995). In the common domain of application of the Saumon et al. (1995) and MHD equations of state, Chabrier \& Baraffe (1997) showed that both developments yield very similar results, which strongly validates the use of the MHD equation of state for our mass range of 0.4 to $1.0 M_{\odot}$.

Despite undeniable advantages of the physical picture, the chemical picture approach leads to smoother thermodynamic quantities, because they can be written as analytical (albeit complicated) expressions of temperature, density and particle abundances. In contrast, the physical picture is normally realized with the unwieldy chemical potential as independent variable, from which density and number abundance follow as dependent quantities. The physical-picture approach involves therefore a numerical inversion before the thermodynamic quantities can be expressed in their "natural" variables temperature, density and particle numbers. This increases computing time greatly, and that is the reason why so far only a limited number of OPAL tables have been produced, only suitable for stars more massive than $\sim 0.8 M_{\odot}$. Therefore we chose MHD for its smoothness, availability, and the possibility to customize it directly for our calculation, despite the in principle - sounder conceptual foundation of OPAL.

\subsection{Opacity tables, treatment of convection, atmosphere and mass loss}

- The OPAL radiative opacities from Iglesias \& Rogers (1996) including the spin-orbit interactions in Fe and relative metal abundances based on Grevesse \& Noels (1993) are used. These tables are completed at low temperatures below $10000 \mathrm{~K}$ with the atomic and molecular opacities by Alexander \& Fergusson (1994).

- We use a value of 1.6 for the mixing length parameter $\alpha$. Various observational comparison support this choice. $\alpha=1.6 \pm 0.1$ leads to the best fit of the red giant branch for a wide range of clusters (see Paper I). It is also the value we get for the calibration of solar models including the same input physics (Richard et al. 1996).

- A grey atmosphere in the Eddington approximation is adopted as boundary condition. Below $\tau=2 / 3$, full integration of the structure equations is performed. We discuss the implications of such an approximation in Sect. 4. 
- Evolution on the pre-main sequence and on the main sequence are calculated at constant mass. On the red giant branch, we take mass loss into account by using the expression by Reimers (1975): $\dot{M}=410^{-13} \eta L$ $R / M$ (in $M_{\odot} \mathrm{yr}^{-1}$ ) where $L, M$ and $R$ are the stellar luminosity, mass and radius respectively (in solar units). At solar metallicity, $\eta=0.5$ is chosen (see Maeder \& Meynet 1989). At $Z=0.001$, the mass loss is lowered by a factor $(0.001 / 0.020)^{0.5}$ with respect to the models at $Z=0.020$ for the same stellar parameters.

\subsection{Nuclear reactions}

- Nuclear reaction rates are due to Caughlan \& Fowler (1988). The screening factors are included according to the prescription of Graboske et al. (1973).

- Deuterium is destroyed on the pre-main sequence at temperatures higher than $10^{6} \mathrm{~K}$ by $D(p, \gamma)^{3} \mathrm{He}$ and, to a lower extent, by $D(D, p)^{3} \mathrm{H}\left(\mathrm{e}^{-} \nu\right)^{3} \mathrm{He}$ and $D(D, n)^{3} \mathrm{He}$. We take into account these three reactions. In order to avoid the follow-up of tritium, we consider the last two reactions as a single process, $D(D, \text { nucl })^{3} \mathrm{He}$. The $\beta$ desintegration is considered as instantaneous, which is justified in view of the lifetime of tritium $\left(\tau_{1 / 2}=12.26 \mathrm{yr}\right)$ compared with the evolutionary timescale. The rate of the $D(D, \text { nucl })^{3} \mathrm{He}$ reaction is written as

$<D D>_{\text {nucl }}=\left(1+\frac{<D D>_{p}}{<D D>_{n}}\right)<D D>_{n}$

where we take for $\frac{\langle D D\rangle_{p}}{\langle D D\rangle_{n}}$ a mean value of 1.065 , in agreement with the rates given by Caughlan \& Fowler (1988). The corresponding mean branch ratios are $I_{p}=$ 0.5157 and $I_{n}=0.4843$.

\subsection{Initial abundances}

- The initial helium content is determined by $Y=$ $0.24+(\Delta Y / \Delta Z) Z$, where 0.24 corresponds to the current value of the cosmological helium (Audouze 1987). We use the value of 3 for the average relative ratio of helium to metal enrichment $(\Delta Y / \Delta Z)$ during galactic evolution. This leads to $(Y, Z)=(0.300,0.020)$ and $(0.243,0.001)$. In addition, computations were also performed with $(Y, Z)=(0.280,0.020)$.

- The relative ratios for the heavy elements correspond to the mixture by Grevesse \& Noels (1993) used in the opacity computations by Iglesias \& Rogers (1996).

- Choosing initial abundance values for $\mathrm{D}$ and ${ }^{3} \mathrm{He}$ is more complex. Pre-solar abundances for both elements have been reviewed in Geiss (1993), however galactic chemical models face serious problems to describe their evolution (see e.g. Tosi 1996), and no reliable prescription exists to extrapolate their values in time. Deuterium is only destroyed by stellar processing since the Big Bang Nucleosynthesis, so that its abundance decreases with time (i.e. with increasing metallicity). On the other hand, the actual contribution of stars of different masses is still subject to a large debate (Hogan 1995; Charbonnel 1995; Charbonnel \& Dias 1998), and observations of $\left({ }^{3} \mathrm{He} / \mathrm{H}\right)$ in the protosolar nebulae (Geiss 1993) and in galactic HII regions present a large dispersion. We adopt the same $(D / H)$ and $\left({ }^{3} \mathrm{He} / \mathrm{H}\right)$ initial values for both metallicities, namely $2.410^{-5}$ and $2.010^{-5}$ respectively.

\subsection{Initial models}

At the low mass range considered in this paper, the observed quasi-static contraction begins rather close to the theoretical deuterium main sequence, once the stars emerge from their parental dense gas and dust. For the present purposes then, we take as starting models polytropic configurations on the Hayashi boundary, neglecting the corrections brought to isochrones and upper tracks by the modern accretion paradigm of star formation (Palla \& Stahler 1993; Bernasconi \& Maeder 1996). For the mass range considered here, these corrections are likely not to exceed $3 \%$ of the Kelvin-Helmholtz timescale for the premain sequence contraction times (Bernasconi 1996). We note, however, that the predicted upper locus for the optical appearance of T Tauri stars in the HR diagram can be as much as half less luminous than the deuterium ignition luminosity on the convective tracks $(\Delta \log L \approx 0.3)$.

\section{Short discussion of the main results}

\subsection{HR diagram and lifetimes}

The HR diagrams for pre-main sequence evolution and for the following phases are given in Figs. 1 and 2 respectively for both metallicities. For each stellar mass, Table 1 displays the lifetimes in the contraction phase and in the deuterium- and hydrogen-burning phases. Note that we did not complete the main sequence evolution computations for the less massive stars which have a H-burning phase longer than the age of the universe; for these stars, our last computed model corresponds to an age of $20 \mathrm{Gyr}$.

- In the stellar mass range we consider, the ignition of deuterium burning (indicated in Fig. 1) takes place in a fully convective interior. During pre-main sequence evolution, a radiative core develops. However a proper radiative branch is absent, since these stars maintain a convective envelope all along contraction until the ZAMS has been reached, and further on.

- From Table 1 one can see that the contraction time lasts less than 4 thousandths of the hydrogen-burning stage. 


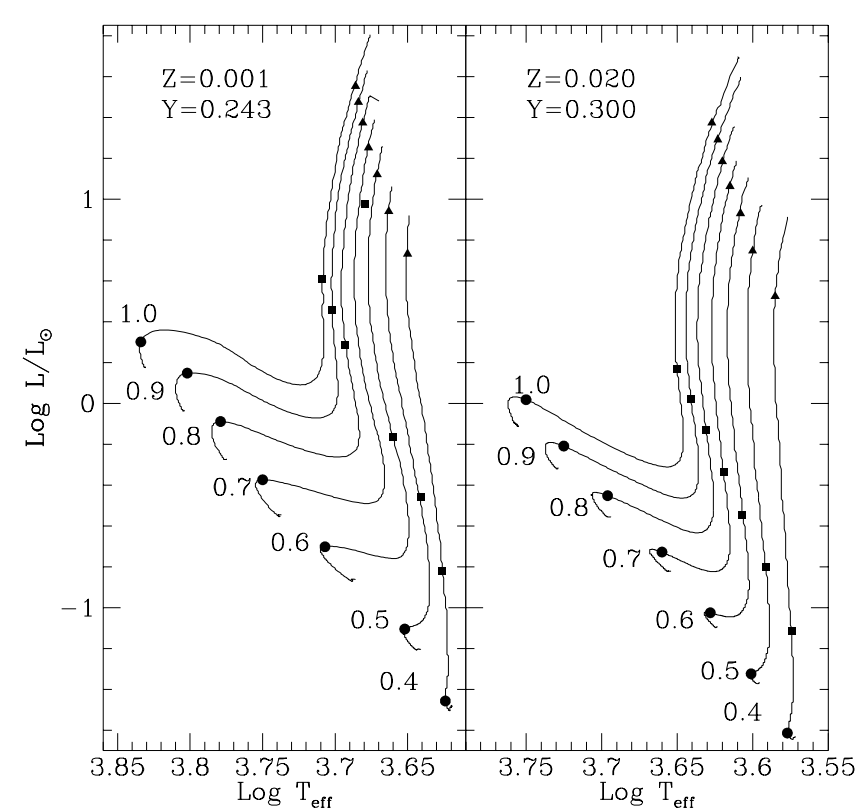

Fig. 1. Theoretical HR diagrams for pre-main sequence evolution for all our models (from 0.4 to $1.0 M_{\odot}$ ) for both metallicities. Triangles mark the ignition of deuterium burning. The radiative core appears at the square location, and the development of a small convective core is indicated by the circles (for $Z=0.020$, the tracks are given only for the models with $Y=0.30$ to avoid confusion)

- Due to opacity effects, the entire evolution (pre-main sequence, main sequence and red giant branch) occurs at higher luminosity and effective temperature for a given stellar mass when the initial metallicity is smaller, or when the hydrogen content is lower for the same initial metallicity.

- As a consequence, the contraction phase and the deuterium- and hydrogen-burning phases for a given stellar mass are shorter at lower metallicity (see Table 1), and at lower hydrogen content for the same value of $Z$.

\subsection{Influence of the equation of state}

When we first compare the results obtained with the MHD and with the simple Geneva equations of state (see Figs. 3 and 4, and Table 2), we obtain essentially the same results than Lebreton \& Däppen (1988). Firstly, the fact that MHD contains $\mathrm{H}_{2}$ molecules, and the simple Geneva code does not, is reflected in a shift essentially along the ZAMS. On the other hand, the Coulomb pressure correction, also contained in MHD, causes a slight shift of the ZAMS, clearly visible for higher masses, where there are no hydrogen molecules in the photosphere. This Coulomb effect has been well discussed in the case of helioseismology (e.g. Christensen-Dalsgaard et al. 1996). Conformal

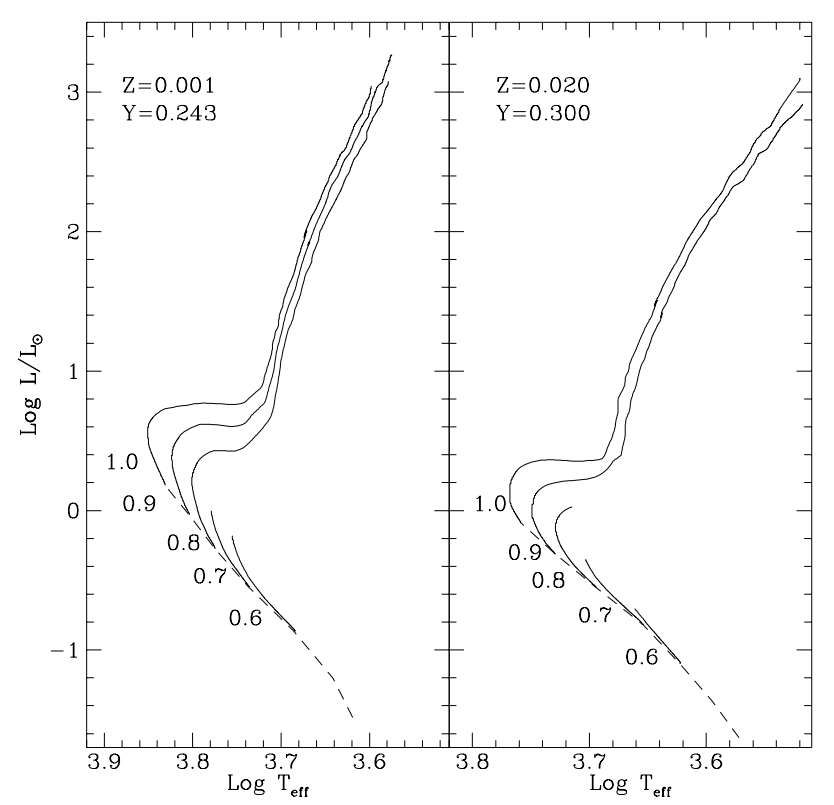

Fig. 2. Theoretical HR diagrams for stellar masses between 0.6 and $1 M_{\odot}$, and position of the zero age main sequence (dashed line) for the entire mass interval

to the effect of the MHD equation of state to push the apparent position on the ZAMS upward, it also decreases the lifetime on the ZAMS (see Table 2).

For comparison, we have computed with the OPAL equation of state two $0.8 M_{\odot}$ models (the lowest mass that can be computed with the current OPAL tables), for both metallicities. As can be seen in Fig. 3, the corresponding tracks are very close to those obtained with the MHD equation of state, the use of the OPAL equation of state leading to slightly higher effective temperature on the ZAMS. As far as their internal structure is concerned, the models computed with MHD equation of state have slightly deeper convection zones. The main sequence lifetime obtained with the MHD equation of state is slightly higher than the one obtained with the OPAL equation of state (Table 2). The comparison shows that down to $0.8 M_{\odot}$ all is fine with the MHD pressure ionization. As mentioned in the introduction, Trampedach \& Däppen (1998) predict a correct functioning of pressure ionization in MHD even for much smaller masses. With the present comparison, we have validated their prediction at least to $0.8 M_{\odot}$.

\section{Comparisons with other sets of models and with observations}

We now compare our models with recent models computed by other groups and with various observations.

The strongest approximation in our models lies in the treatment of the atmosphere and of the surface boundary 


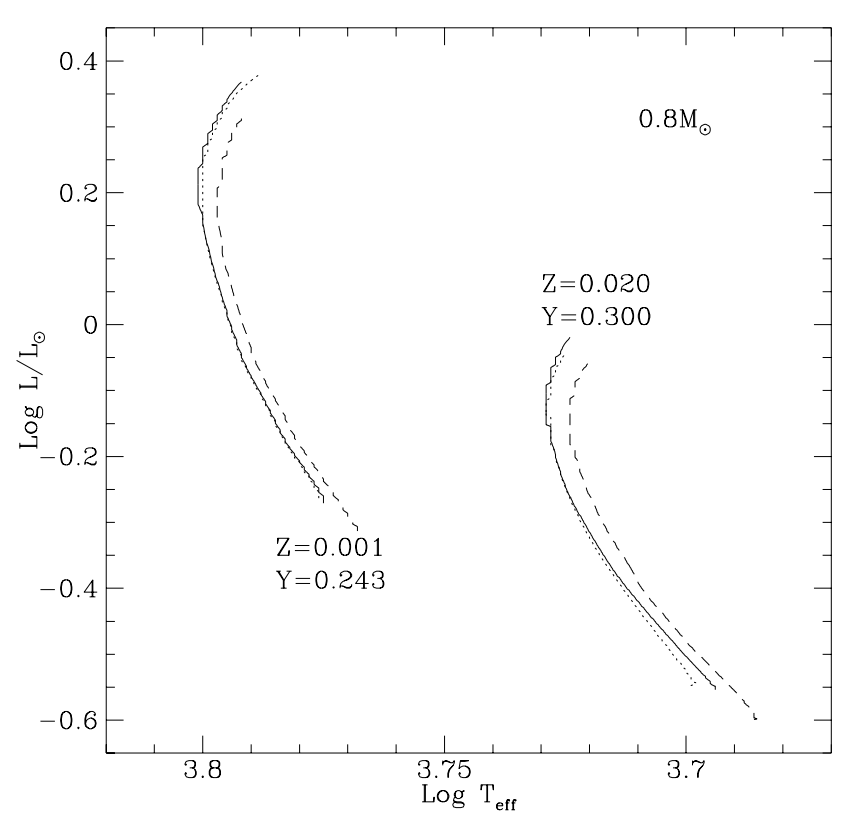

Fig. 3. Influence of the MHD equation of state on the main sequence evolutionary track of the $0.8 M_{\odot}$ models. The solid, dotted and dashed lines correspond to models computed with the MHD, OPAL and the simple Geneva (Sect. 1) equations of state, respectively

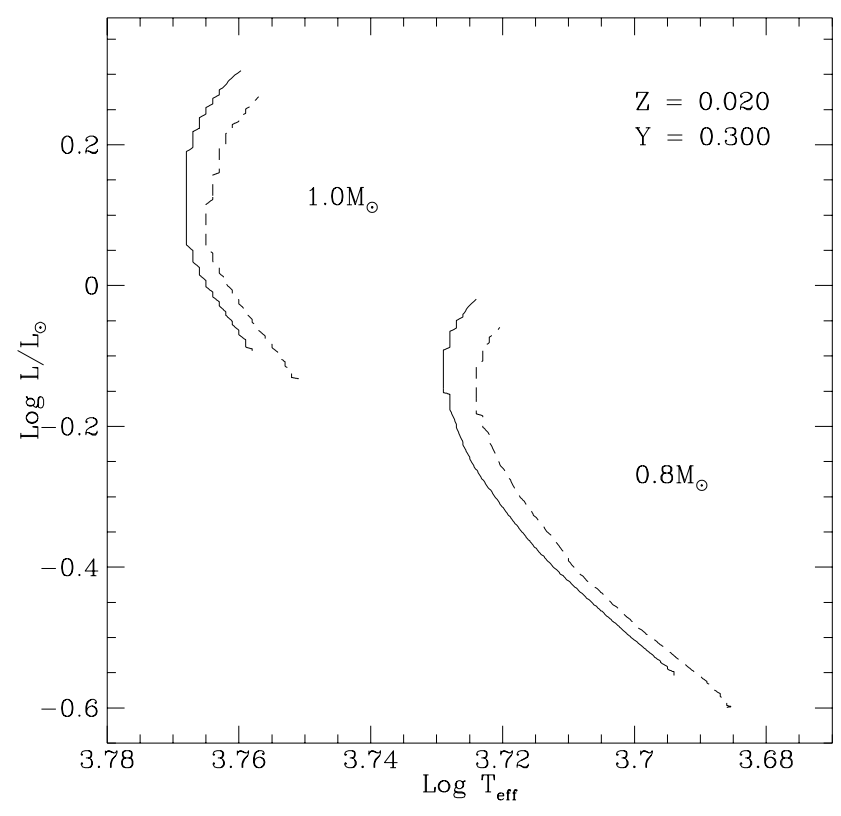

Fig. 4. Influence of the MHD equation of state on the main sequence evolutionary track of the 1.0 and $0.8 M_{\odot}$ models for $Z=0.020$. The full and dashed lines correspond to models computed with the MHD and with the simple Geneva equations of state, respectively
Table 1. Lifetimes in contraction and nuclear phases (in units of $10^{6} \mathrm{yr}$ ), and ratio of the contraction time $t_{\mathrm{c}}$ to the hydrogen burning time $t_{\mathrm{H}}$. For the less massive stars which have a main sequence phase longer than the Hubble time, we stopped the computations at an age of $20 \mathrm{Gyr}$

\begin{tabular}{|c|c|c|c|c|c|}
\hline $\begin{array}{l}Z \\
Y\end{array}$ & $\begin{array}{l}\text { Initial } \\
\text { mass }\end{array}$ & $\begin{array}{c}\text { Contraction } \\
\text { phase }\end{array}$ & $\begin{array}{l}\text { D-burning } \\
\text { phase }\end{array}$ & $\begin{array}{l}\text { H-burning } \\
\text { phase }\end{array}$ & $t_{\mathrm{c}} / t_{\mathrm{H}}$ \\
\hline 0.001 & 0.4 & 160.10 & 0.417 & - & - \\
\hline 0.243 & 0.5 & 81.11 & 0.321 & - & - \\
\hline . & 0.6 & 51.89 & 0.277 & - & - \\
\hline . & 0.7 & 36.44 & 0.240 & - & - \\
\hline . & 0.8 & 31.20 & 0.214 & 14338.03 & 0.0022 \\
\hline . & 0.9 & 22.82 & 0.196 & 9051.01 & 0.0025 \\
\hline . & 1.0 & 19.34 & 0.187 & 6847.20 & 0.0028 \\
\hline 0.020 & 0.4 & 153.84 & 0.605 & - & - \\
\hline 0.300 & 0.5 & 117.34 & 0.476 & - & - \\
\hline . & 0.6 & 88.26 & 0.389 & - & - \\
\hline . & 0.7 & 68.80 & 0.330 & - & - \\
\hline . & 0.8 & 58.15 & 0.302 & 22713.11 & 0.0026 \\
\hline . & 0.9 & 44.30 & 0.276 & 14070.96 & 0.0031 \\
\hline . & 1.0 & 32.80 & 0.252 & 9059.52 & 0.0036 \\
\hline 0.020 & 0.4 & 161.79 & 0.640 & - & - \\
\hline 0.280 & 0.5 & 124.50 & 0.484 & - & - \\
\hline . & 0.6 & 93.75 & 0.407 & - & - \\
\hline . & 0.7 & 72.86 & 0.344 & - & - \\
\hline . & 0.8 & 62.34 & 0.309 & 26372.72 & 0.0024 \\
\hline . & 0.9 & 48.26 & 0.286 & 16421.94 & 0.00002 \\
\hline . & 1.0 & 37.98 & 0.263 & 10662.62 & 0.00002 \\
\hline
\end{tabular}

Table 2. Lifetimes on the main sequence (in units of $10^{6} \mathrm{yr}$ ) for models computed with the MHD, the OPAL and the simple Geneva equation of state

\begin{tabular}{ccccc}
\hline$Z$ & $M / M_{\odot}$ & $t_{\mathrm{H}}(\mathrm{MHD})$ & $t_{\mathrm{H}}(\mathrm{OPAL})$ & $t_{\mathrm{H}}($ orig. Geneva $)$ \\
\hline & & & & \\
0.020 & 1.0 & 9059.5 & 8968.1 & 9970.1 \\
0.020 & 0.8 & 22713.3 & 22361.4 & 25151.3 \\
0.001 & 0.8 & 14338.0 & 13986.0 & \\
\hline
\end{tabular}

conditions, which are specified by the Eddington approximation. In Figs. 5 and 6 we compare our results with the models of D'Antona \& Mazzitelli (1994) and Tout et al. (1996) which also rely on a simple treatment of the boundary conditions. For the mass range considered in our work we obtain predictions very similar to D'Antona \& Mazzitelli.

Sophisticated model atmospheres for the computation of very low mass stars have been developed recently (Baraffe et al. 1995, 1998; Brett 1995; Allard et al. 1997). Their use becomes crucial for very low mass stars 


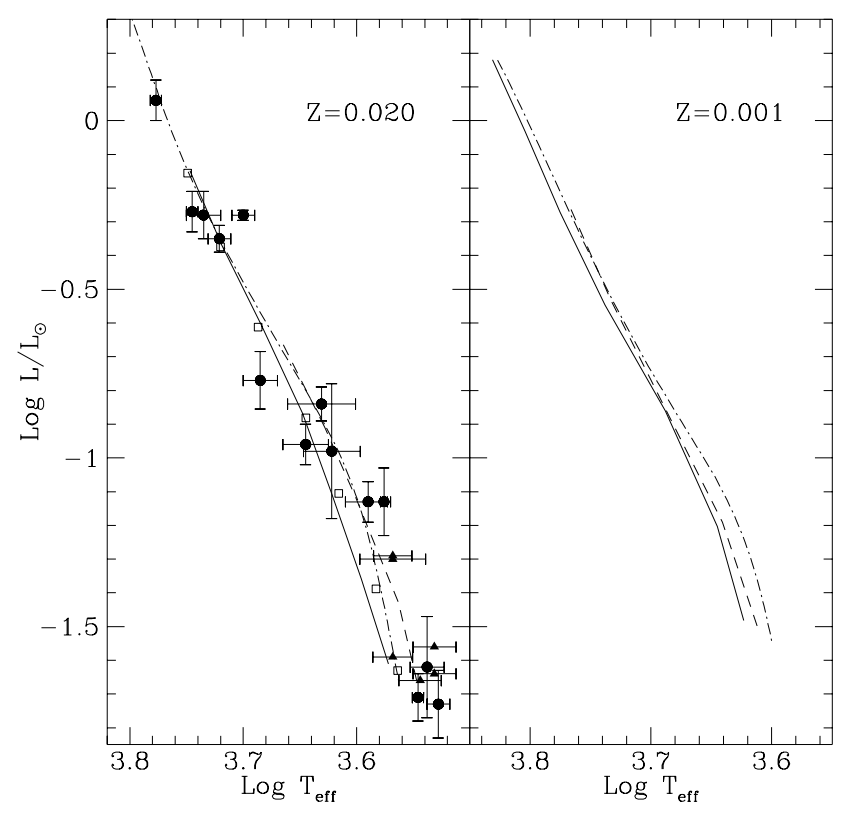

Fig. 5. Theoretical HR diagrams at two values of the metallicity. Solid lines: our models $(Y=0.28$ for $Z=0.02)$; dashed lines: Chabrier \& Baraffe (1997); dashed-dotted lines: Tout et al. (1997); open squares: D'Antona \& Mazzitelli (1994). The models are taken at $0.1 \mathrm{Gyr}$, except for the zams models of T97. The observational data are from Popper (1980; black points) and from Leggett et al. (1996; black triangles)

$\left(M \lesssim 0.4 M_{\odot}\right)$ down to the brown dwarf limit. We refer to Chabrier \& Baraffe (1997) and Baraffe et al. (1998) for a discussion of the physical basis of the differences. As can be seen in Fig. 5, the Eddington approximation we use results in higher $T_{\text {eff }}$ (from 2 to $5 \%$ depending on the metallicity) compared to the Chabrier \& Baraffe (1997) models at our low mass end; our models also have slightly smaller radius (see Fig. 6). In this mass range the predictions agree well with the observations of Popper (1980) and Leggett et al. (1996) and do not allow to disregard one model with respect to the other.

In Fig. 7 we show the predicted mass-luminosity relations for the $V$ and $K$ band for different metallicities and ages. The magnitudes were derived from the standard stellar library for evolutionary synthesis of Lejeune et al. (1998), which provides empirically calibrated colours for solar metallicity and a semi-empirical correction for nonsolar metallicities. The comparison with the observations of Andersen (1991) and Henry \& McCarthy (1993) shows a good agreement with our predictions for both the $V$ and $K$ band (Fig. 7). Again a similar agreement is obtained with the models of Brocato et al. (1998) and Baraffe et al. (1998).

From the comparisons in Figs. 5 to 8, we conclude (in agreement with Alexander et al. 1997) that for the mass range considered in this work an approximate

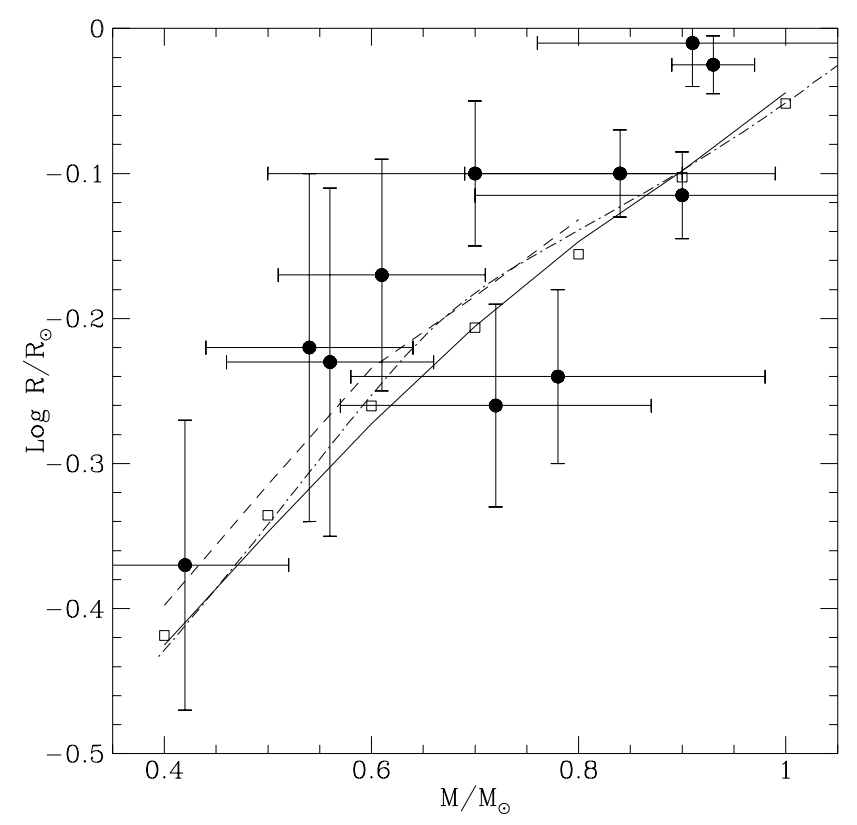

Fig. 6. Dependence of the stellar radius on mass for solar metallicity models. See Fig. 5 for the references of the models and observations

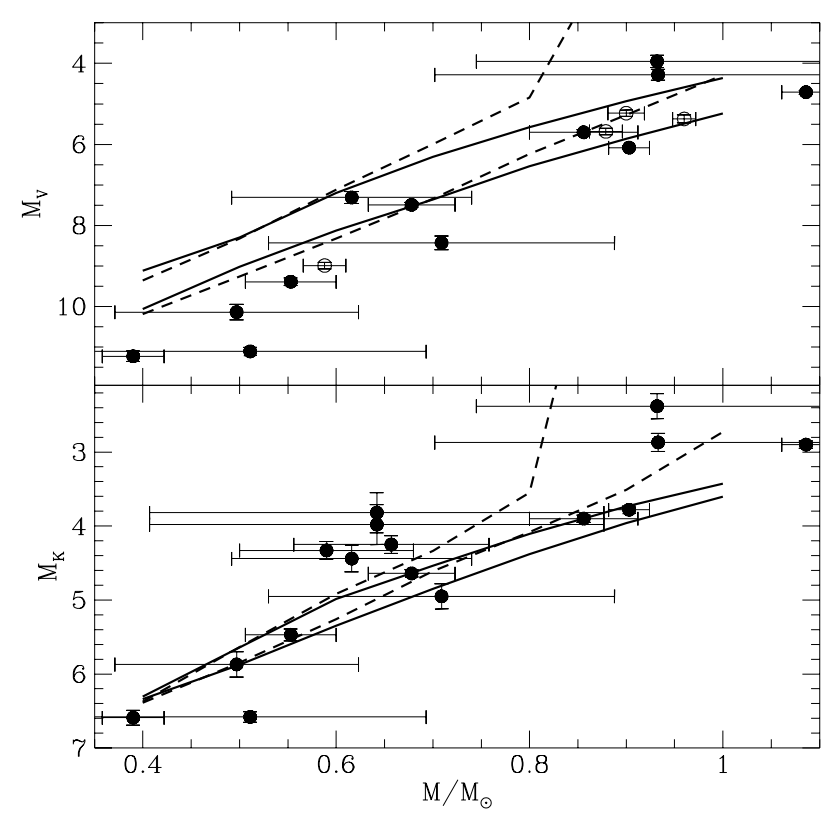

Fig. 7. Mass-luminosity relations at 0.1 and $10 \mathrm{Gyr}$ (solid and dashed lines respectively) for the solar (with $Y=0.28$ ) and low metallicity models (lower and upper curves respectively). The observations are from Andersen (1991) and Henry \& McCarthy (1993) (white and black circles respectively) 
treatment of the stellar atmosphere leads to a satisphactory agreement between theoretical predictions and observations. Independently ab initio stellar interior and atmosphere models allowing the detailed predictions of all observational properties are of fundamental importance for our understanding of very low mass stars which are out of the scope of the present grids.

\section{Appendix A: Appendix: How to obtain the tables by file-transfer}

The results of this work and of our previous grids (Papers I to VI) are published by Astronomy and Astrophysics at the Centre de Données Stellaires (CDS at Strasbourg) where the corresponding tables are available in electronic form:

http://cdsweb.u-strasbg.fr. These data can also be obtained from the Geneva Observatory

http: //obswww. unige.ch/

(contact Corinne. Charbonnel@obs-mip.fr).

An ensemble of models were selected to describe each evolutionary track. For each model the tables display the age, actual mass, $\log L / L_{\odot}, \log T_{\text {eff }}$, the surface abundances in mass fraction of $\mathrm{H},{ }^{4} \mathrm{He},{ }^{12} \mathrm{C},{ }^{13} \mathrm{C},{ }^{14} \mathrm{~N},{ }^{16} \mathrm{O}$, ${ }^{17} \mathrm{O},{ }^{18} \mathrm{O},{ }^{20} \mathrm{Ne},{ }^{22} \mathrm{Ne}$, the core mass fraction Qcc, $\log (-\dot{M})$ (where $\dot{M}$ is the mass loss rate on the red giant branch), $\log \rho_{\mathrm{c}}$ (where $\rho_{\mathrm{c}}$ is the central density), $\log T_{\mathrm{c}}$ (where $T_{\mathrm{c}}$ is the central temperature), and the central abundances in mass fraction of the above elements. A detailed description of the models selection and of the tables contents is given in Paper I. We now also provide photometric data in verious systems for all tracks and isochrones (see Schaerer \& Lejeune 1998).

Acknowledgements. We thank Thibault Lejeune for calculations prior to publication. C.C. wishes to express her gratitude to the staff of the Space Telescope Science Institute where this study was carried out. W.D. was supported in part by the grants AST-9315112 and AST-9618549 of the National Science Foundation. D.S. is supported by the Swiss National Foundation for Scientific Research and acknowledges partial support from the Directors Discretionary Research Fund of the STScI.

\section{References}

Alexander D.R., Fergusson J.W., 1994, ApJ 437, 879

Alexander D.R., Brocato E., Cassisi S., Castellani V., Ciacio F., Degl'Innocenti S., 1997, A\&A 317, 90

Allard F., Hauschildt P.H., Alexander D.R., Starrfield S., 1997, ARA\&A 35, 137

Andersen J., 1991, A\&AR 3, 91

Audouze J., 1987, in Observational Cosmology, IAU Symp. 124, Hewitt A. et al. (eds.). Reidel Publ., p. 89

Baraffe I., Chabrier G., Allard F., Hauschildt P.H., 1995, ApJ 446, L35
Baraffe I., Chabrier G., Allard F., Hauschildt P.H., 1998, A\&A (submitted) (astro-ph/9805009)

Basu S., Christensen-Dalsgaard J., 1997, A\&A 322, L5

Bernasconi P.A., 1996, A\&AS 120, 57

Bernasconi P.A., Maeder A., 1996, A\&A 307, 829

Brett J.M., 1995, A\&A 295, 736

Brocato E., Cassisi S., Castellani V., 1998, MNRAS 295, 711

Caughlan G.R., Fowler W.A., 1988, Atomic Data Nuc. Data Tabs. 40, 283

Chabrier G., Baraffe I., 1997, A\&A 327, 1039

Charbonnel C., 1995, ApJ 453, L41

Charbonnel C., Dias do Nascimento J., 1998, A\&A 336, 915

Charbonnel C., Lebreton Y., 1993, A\&A 280, 666

Charbonnel C., Meynet G., Maeder A., Schaller G., Schaerer D., 1993, A\&A 102, 339

Charbonnel C., Meynet G., Maeder A., Schaerer D., 1996, A\&AS 115, 339

Christensen-Dalsgaard J., Däppen W., Lebreton Y., 1988, Nat 336,634

Christensen-Dalsgaard J., Däppen W., and the GONG team, 1996, Sci 272,1286

D'Antona F., Mazzitelli I., 1994, ApJS 90, 467

D'Antona F., Mazzitelli I., 1996, ApJ 456, 329

Däppen W., Mihalas D., Hummer D.G., Mihalas B.W., 1988, ApJ 332, 261

Ebeling W., Kraeft W.D., Kremp D., 1976, Theory of Bound States and Ionization Equilibrium in Plasmas and Solids. Akademie Verlag, Berlin, DDR

Geiss J., 1993, Origin and Evolution of the Elements, Prantzos N., Vangioni-Flam E., Cassé M. (eds.). Cambridge University Press, p. 89

Graboske H.C., de Witt H.E., Grossman A.S., Cooper M.S., 1973, ApJ 181, 457

Grevesse N., Noels A., 1993, in: "Origin and Evolution of the Elements", Prantzos N., Vangioni-Flam E., Cassé M. (eds.), CUP

Henry T.J., McCarthy D.W., 1993, AJ 106, 773

Hogan C., 1995, ApJ 441, L17

Hummer D.G., Mihalas D., 1988, ApJ 331, 794

Iglesias C.A., Rogers F.J., 1996, ApJ 464, 943

Lebreton Y., Däppen W., 1988, Seismology of the Sun and the Sun-like Stars, Domingo V., Rolfe E.J. (eds.). ESA SP-286, 661

Leggett S.K., Allard F., Berriman G., Dahn C.C., Hauschildt P.H., 1996, ApJS 104, 117

Lejeune T., Cuisinier F., Buser R., 1997, A\&AS (in press) (astro-ph/9710350)

Maeder A., Meynet G., 1989, A\&A 210, 155

Meynet G., Maeder A., Schaller G., Schaerer D., Charbonnel C., 1994, A\&AS 103, 97

Mihalas D., Hummer D.G., Däppen W., 1988, ApJ 331, 815

Mowlavi N., Schaerer D., Meynet G., Bernasconi P.A., Maeder A., Charbonnel C., 1998, A\&AS 128, 1

Palla F., Stahler S.W., 1993, ApJ 418, 414

Popper D.M., Ann. Rev. Astr. Ap. 18, 115

Reimers D., 1975, Mem. Soc. Roy. Sci. Liège 8, 369

Richard O., Vauclair S., Charbonnel C., Dziembowski W.A., 1996, A\&A 312, 1000

Rogers F.J., 1986, ApJ 310, 723

Rogers F.J., Swenson F.J., Iglesias C.A., 1996, ApJ 456, 902

Saumon D., Chabrier G., 1991, Phys. Rev. A44, 5122 
Saumon D., Chabrier G., 1992, Phys. Rev. A46,

Saumon D., Chabrier G., VanHorn H.M., 1995, ApJS 99, 713

Schaerer D., Lejeune T., 1998 (in preparation)

Schaerer D., Meynet G., Maeder A., Schaller G., 1992, A\&AS 98, 523

Schaerer D., Charbonnel C., Meynet G., Maeder A., Schaller G., 1993, A\&AS 102, 339

Schaller G., Schaerer D., Meynet G., Maeder A., 1992, A\&AS 96, 269

Seaton M., 1987, J. Phys. B: Atom. Molec. Phys. 20, 6363
Seaton M.J., 1992, in: Astrophysical Opacities, Mendoza C. \& Zeippen C. (eds.), Revista Mexicana de Astronomía y Astrofísica, p. 180

Tosi M., 1996, From Stars to Galaxies: The Impact of Stellar Physics on Galaxy Evolution, ASP Conf. Series, Vol. 78, Leitherer C., Fritze U. - von Alvensleben, Huchra J. (eds.), p. 299

Tout C.A., Pols O., Eggleton P.P., Han Z., 1996, MNRAS 281, 257

Trampedach R., Däppen W., 1998, A\&A (submitted) 